TRANSACTIONS OF THE

AMERICAN MATHEMATICAL SOCIETY

Volume 196, 1974

\title{
ANY INFINITE-DIMENSIONAL FRÉCHET SPACE HOMEOMORPHIC WITH ITS COUNTABLE PRODUCT IS TOPOLOGICALLY A HILBERT SPACE
}

BY

\author{
WESLEY E. TERRY
}

ABSTRACT. In this paper we will prove that any infinite-dimensional Fréchet space homeomorphic with its own countable product is topologically a Hilbert space. This will be done in two parts. First we will prove the result for infinite-dimensional Banach spaces, and then we will show that the result for Fréchet spaces follows as a corollary.

1. Introduction. Let $F$ be a Fréchet space (complete locally convex metric topological vector space) such that $F$ is homeomorphic with $(\cong)$ its own countable product $\left(F^{\omega}\right)$. In the following we will show that such a Fréchet space is homeomorphic with a Hilbert space of appropriate weight.

In an addendum to [13], Toruniczyk claims a proof of the same result. The two proofs are independent and use techniques which are completely different.

2. Preliminaries. Let $\Lambda$ be a set of cardinality $x$. The space $l_{p}(x)$ for fixed $p \geq 1$ is defined to be the set of all real functions $r=\left\{r_{\lambda}\right\}$ defined on the set $\Lambda$ with at most a countable number of nonzero elements and with $\Sigma_{\lambda}\left|r_{\lambda}\right|^{p}<\infty$. The norm on $l_{p}(x)$ is $\|r\|=\left\{\Sigma_{\lambda}\left|r_{\lambda}\right|^{p}\right\}^{1 / p}$. When $p=2$, this is a Hilbert space of weight $x$.

In [1] Bessaga has proven the following theorem:

Theorem 1. If $F$ is a Fréchet space then $l_{1}(w F) \cong l_{1}(w F) \times F$ where $w F$ is the cardinal equal to the weight of $F$.

Proof. See 8.1, 3.2, 8.4, and 8.5 in [1].

We will use the existence of such a homeomorphism for a Banach space to show $B \simeq B^{\omega}$ implies $B \simeq l_{1}(w B)$.

We will now prove an imbedding theorem for Banach spaces.

Received by the editors April 27, 1973.

AMS (MOS) subject classifications (1970). Primary 46A05, 46B05, 46C05; Secondary $57 \AA 17,57 \AA 20$.

Key words and phrases. Hilbert space, infinite-dimensional Fréchet space, countable product, Z-set, negligible.

Copyrimt $\odot$ 1974, American Mathematical Society 
Lemma 2. Let $B$ be an infinite-dimensional Banach space. Then there is a closed imbedding of $l_{1}(w B)$ into $B^{\omega}$.

Proof. Fix $1 / n$ with $n$ a positive integer, and let $\left\{U_{\alpha}^{\prime}\right\}_{a \in A}$ be the collection of all $1 / n$ balls of $l_{1}(w B)$. Find a locally finite refinement $\left\{U_{\beta}\right\}_{\beta \in B}$ and let $\left\{\psi_{\beta}\right\}_{\beta \in \mathrm{B}}$ be a partition of unity subordinate to this cover. Applying a theorem of Michael (Lemma 2.1(c) and (e) of [11]) we may obtain a locally finite refinement $\left\{G_{i \beta}^{n}\right\}_{\beta \in E_{i}^{n}}, i=1,2, \cdots$, such that $G_{i \beta} \cap G_{i \gamma}=\varnothing$ if $\beta \neq \gamma$. Do this for each positive integer. Next, by Lemma 1.2 of [4], pick a collection of disjoint open sets in the unit sphere of $B$ having cardinality the weight of $B$, and pick one point $x_{\beta}$ from each of the open sets.

Define

$$
\begin{aligned}
g: l_{1}(w B) & \rightarrow \prod_{n=1}^{\infty}\left(\prod_{i=1}^{\infty} B_{i}\right)_{n}, \\
\left\{r_{\beta}\right\} & \mapsto\left\{\sum_{i \beta \in B_{i}^{n}} \psi_{i \beta}^{n}\left(r_{i \beta}\right) x_{i \beta}\right\} .
\end{aligned}
$$

Note that there is at most one nonzero coordinate in each $B_{i}$ for each $n$ since a point may lie in at most one subset of a disjoint collection.

Now, $g$ is clearly continuous, and $g$ is one-to-one since no point is within $1 / n$ of any other point for all $n$. To see $g^{-1}$ is continuous, observe that given $\epsilon>0$ and a sequence $\left\{y^{j}\right\}$ converging to $y$ in the image of $g$, we may pick $n$ such that $2 / n<\epsilon_{0}$. Then pick a $k$ such that $y$ has a nonzero coordinate in $B_{k, n}$ where $B_{k, n}$ is the $k$ th copy of $B$ in the $n$th product $\left(\Pi_{i=1}^{\infty} B_{i}\right)_{n}$. Let $x_{k \beta}$ be a point with the nonzero real multiple. Then since convergence in a product is equivalent to coordinate-wise convergence, pick $J$ such that $j>J$ implies the $x_{k \beta}$ multiple in $B_{k, n}$ is nonzero for $y^{j}$. It is here that we are using the fact that $\left\{x_{\beta}\right\}$ is a discrete set. Then $g^{-1}\left(y^{j}\right)$ and $g^{-1}(y)$ are contained in a $1 / n$ ball in $l_{1}(w B)$ for all $j>J$. Therefore, the distance between $g^{-1}\left(y^{j}\right)$ and $g^{-1}(y)$ is less than $\epsilon$ for all $j>J$.

This gives an imbedding of $l_{1}(w B)$ as a $G_{\delta}$ set in $B^{\omega}$. Now, given a Banach space $B$, by the Hahn-Banach theorem $B \simeq \mathrm{R} \times N$ where $\mathrm{R}$ is a copy of the reals. Therefore,

$$
B^{\omega} \simeq(\mathbf{R} \times N)^{\omega} \cong \mathbf{R}^{\omega} \times N^{\omega} \cong \mathbf{R}^{\omega} \times B^{\omega}
$$

Using this fact, we may imbed this $G_{\delta}$ as a closed subset of $B^{\omega}$. (See Kuratowski [9, pp. 229 and 430].) Thus we obtain a closed imbedding.

Remark 1. The proof of Lemma 2 may be adapted to any open cone, $M$, which 
is a topological vector space. All we need to do is pick a discrete set $\left\{x_{\beta}\right\}$ in $M^{\omega}$ which is radially independent and for which the cardinality of $\left\{x_{\beta}\right\}$ equals the weight of $M$. See Lemma 1.2 of [4] to see that we may pick a discrete set $\left\{x_{\beta}\right\}$ chosen from disjoint open sets. In the nonseparable case, using a Zorn's lemma argument together with the fact that the topology of a ray is second countable, we may then produce a radially independent set. The separable case uses the fact that any metric topological vector space has a real factor. See Henderson's paper [ 5 ] for theorems on spaces being open cones.

Given $\left\{\left(B_{i},\|\|_{i}\right) \mid i=1,2, \cdots\right\}$, a collection of Banach spaces, and given $l_{1}=l_{1}\left(x_{0}\right)$, we will define $\Sigma_{l_{1}} B_{i}$ to be the set of sequences $\left\{x_{i}\right\}, x_{i} \in B_{i}$, such that $\left\{\left\|x_{i}\right\|_{i}\right\} \in l_{1}$. If $\left(B_{i},\|\|_{i}\right)$ is the same pair for each $i$, we will just write $\Sigma_{l_{1}} B$. It is easy to show that $\Sigma_{l_{1}} B$ is a Banach space.

Lemma 3. Given an infinite-dimensional Banach space $B$, there is a bomeomorphism

$$
\begin{aligned}
b: & \Sigma_{l_{1}}\left(l_{1} \backslash\{0\}\right) \times \Sigma_{l_{1}} l_{1}(w B) \\
& \rightarrow \Sigma_{l_{1}}\left(l_{1} \backslash\{0\}\right) \times \Sigma_{l_{1}} B \times \Sigma_{l_{1}}\left(l_{1} \backslash\{0\}\right) \times \Sigma_{l_{1}} l_{1}(w B)
\end{aligned}
$$

satisfying the following properties:

(1) $b(r, 0)=\left(\psi_{1}(r), 0, \psi_{2}(r), 0\right)$ where $\psi: \Sigma_{l_{1}}\left(l_{1} \backslash\{0\}\right) \rightarrow \Sigma_{l_{1}}\left(l_{1} \backslash\{0\}\right) \times$ $\Sigma_{l_{1}}\left(l_{1} \backslash\{0\}\right)$ is the isomorphism sending odd coordinates to the first copy and even coordinates to the second copy and where $\psi_{i}$ is projection of $\psi$ into the ith copy of $\Sigma_{l_{1}}\left(l_{1} \backslash\{0\}\right), i=1,2$.

(2) For each positive integer $n$ there is a positive integer $m$ such that

$$
b_{4} \circ \underbrace{\left(b_{3}, b_{4}\right) \circ \ldots \circ\left(b_{3}, b_{4}\right)}_{m}(r, x)=0
$$

for all $x$ with at most the first $n$ coordinates nonzero. Here $b_{i}$ is projection of $b$ onto the ith coordinate.

(3) $|r|_{l_{1}}+|x|_{w}=\left|b_{1}(r, x)\right|_{l_{1}}+\left|b_{2}(r, x)\right|_{B}+\left|b_{3}(r, x)\right|_{l_{1}}+\left|b_{4}(r, x)\right|_{w}$ where $b_{i}$ is again projection of $b$ onto the ith coordinate and where ||$_{l_{1}},||_{B}$, and ||$_{w}$ are the norms on the spaces $\Sigma_{l_{1}} l_{1}, \Sigma_{l_{1} B}$ and $\Sigma_{l_{1}} l_{1}(w B)$ respectively.

Proof. $\Sigma_{l_{1}} l_{1}(w B)=l_{1}(w B)$. Therefore, Theorem 1 guarantees a homeomorphism $g^{\prime}: \Sigma_{l_{1}} l_{1}(w B) \rightarrow \Sigma_{l_{1}} B \times \Sigma_{l_{1}} l_{1}(w B)$. It was shown by Klee in [G] and [7] that the unit sphere of any infinite-dimensional Banach space $B^{\prime}$ is homeomorphic with any of its hyperplanes (subspace of deficiency one). Thus $B^{\prime}=\mathbf{R} \times N$ and the unit sphere is homeomorphic with $N$. But any infinite-dimensional Banach space has an $l_{1}$ factor. (See $[12$, Remark 1].) Thus $N \cong \mathbf{R} \times N$, and the unit 
sphere of $B^{\prime}$ is homeomorphic with $B^{\prime}$. Therefore, given $g^{\prime}: \Sigma_{l_{1}} l_{1}(w B) \rightarrow \Sigma_{l_{1}} B \times$ $\Sigma_{l_{1}} l_{1}(w B)$ there is a homeomorphism $g^{*}$ from the unit sphere of $\Sigma_{l_{1}} l_{1}(w B)$ to the unit sphere of $\Sigma_{l_{1}} B \times \Sigma_{l_{1}} l_{1}(w B)$ under norm ||$_{B}+\mid l_{w}$. But then there is a radial homeomorphism

$$
\begin{aligned}
g: \Sigma_{l_{1}} l_{1}(w B) & \rightarrow \Sigma_{l_{1}} B \times \Sigma_{l_{1}} l_{1}(w B), \\
x & \mapsto\left(|x|_{w} g_{1}^{*}\left(\frac{x}{|x|_{w}}\right),|x|_{w} g_{2}^{*}\left(\frac{x}{|x|_{w}}\right)\right) . \\
g^{-1}: \Sigma_{l_{1}} B \times \Sigma_{l_{1}} l_{1}(w B) & \rightarrow \Sigma_{l_{1}} l_{1}(w B), \\
(y, z) & \mapsto\left(|y|_{B}+|z|_{l_{1}}\right) g^{*-1}\left(\frac{y}{|y|_{B}+|z|_{l_{1}}}, \frac{z}{|y|_{B}+|z|_{l_{1}}}\right) .
\end{aligned}
$$

In both cases zero is sent to zero. Now $g$ has the property that $g^{-1}\left(\Sigma_{l_{1}} B \times\{0\}\right)$ is a radial subset of $l_{1}(w B)$.

Now, by a theorem of the author's [12], $B^{\omega} \cong \Sigma_{l_{1}} B$ for all infinite-dimensional Banach spaces. Therefore, by Lemma 2, there is a closed imbedding $f$ of $\Sigma_{l_{1}} l_{1}(w B)$ into $\Sigma_{l_{1}} B \times\{0\} \subset \Sigma_{l_{1}} B \times \Sigma_{l_{1}} l_{1}(w B)$. Define the map

$$
\begin{aligned}
& \Sigma_{l_{1}} l_{1}(w B) \times\{0\} \stackrel{\left(s^{-10} f\right) \times \mathrm{id}}{\longrightarrow} \Sigma_{l_{1}} l_{1}(w B) \times\{0\} \\
& n \\
& \Sigma_{l_{1}} l_{1}(w B) \times \Sigma_{l_{1}} l_{1}(w B) \quad \Sigma_{l_{1}} l_{1}(w B) \times \Sigma_{l_{1}} l_{1}(w B) .
\end{aligned}
$$

This is a closed imbedding, and therefore, by a theorem of Klee [8], there is a homeomorphism

$$
G: \Sigma_{l_{1}} l_{1}(w B) \times \Sigma_{l_{1}} l_{1}(w B) \rightarrow \Sigma_{l_{1}} l_{1}(w B) \times \Sigma_{l_{1}} l_{1}(w B)
$$

which extends this map.

Let $\phi: \Sigma_{l_{1}} l_{1}(w B) \rightarrow \Sigma_{l_{1}} l_{1}(w B) \times \Sigma_{l_{1}} l_{1}(w B)$ be the isomorphism sending odd coordinates to the first copy and even coordinates to the second. Define

$$
b^{\prime}: \Sigma_{l_{1}} l_{1}(w B) \rightarrow \Sigma_{l_{1}} B \times \Sigma_{l_{1}} l_{1}(w B)
$$

by

$$
\begin{aligned}
b^{\prime}(x)= & \left(\mathrm{id} \times \phi^{-1}\right) \circ\left(\mathrm{id} \times \phi^{-1} \times \mathrm{id}\right) \circ(\mathrm{id} \times G \times \mathrm{id}) \circ\left(\mathrm{id} \times \phi^{-1} \times \mathrm{id} \times \mathrm{id}\right) \\
& \circ(g \times \mathrm{id} \times \mathrm{id} \times \mathrm{id}) \circ(\phi \times \phi) \circ \phi(x) .
\end{aligned}
$$

That is 


$$
\begin{aligned}
& \Sigma_{l_{1} l_{1}(w B) \longrightarrow}^{\longrightarrow} \Sigma_{l_{1} l_{1}(w B) \times \Sigma_{l_{1}} l_{1}(w B)} \\
& \stackrel{\phi \times \phi}{\longrightarrow} \Sigma_{l_{1}} l_{1}(w B) \times \Sigma_{l_{1}} l_{1}(w B) \times \Sigma_{l_{1}} l_{1}(w B) \times \Sigma_{l_{1}} l_{1}(w B) \\
& \stackrel{\text { gxidxidxid }}{\longrightarrow} \Sigma_{l_{1}} B \times \Sigma_{l_{1}} l_{1}(w B) \times \Sigma_{l_{1}} l_{1}(w B) \times \Sigma_{l_{1}} l_{1}(w B) \times \Sigma_{l_{1}} l_{1}(w B) \\
& \stackrel{\mathrm{id} \times \phi^{-1} \times \mathrm{idxid}}{\longrightarrow} \Sigma_{l_{1}} B \times \Sigma_{l_{1}} l_{1}(w B) \times \Sigma_{l_{1}} l_{1}(w B) \times \Sigma_{l_{1}} l_{1}(w B) \\
& \stackrel{\mathrm{id} \times G \times \mathrm{id}}{\longrightarrow} \Sigma_{l_{1}} B \times \Sigma_{l_{1}} l_{1}(w B) \times \Sigma_{l_{1}} l_{1}(w B) \times \Sigma_{l_{1}} i_{1}(w B) \\
& \stackrel{\mathrm{id} \times \phi^{-1} \times \mathrm{id}}{\longrightarrow} \Sigma_{l_{1}} B \times \Sigma_{l_{1}} l_{1}(w B) \times \Sigma_{l_{1}} l_{1}(w B) \\
& \stackrel{\mathrm{id} \times \phi^{-1}}{\longrightarrow} \Sigma_{l_{1}} B \times \Sigma_{l_{1}} l_{1}(w B) \text {. }
\end{aligned}
$$

Since $b^{\prime}$ is a composition of homeomorphisms, $b^{\prime}$ is a homeomorphism.

Finally, define a new homeomorphism $b$ by

$$
\begin{gathered}
\left.b: \Sigma_{l_{1}}\left(l_{1} \backslash\{0\}\right) \times \Sigma_{l_{1}} l_{1}(w B) \rightarrow \Sigma_{l_{1}}\left(l_{1} \backslash\{0\}\right) \times \Sigma_{l_{1}} B \times \Sigma_{l_{1}}\left(l_{1} \backslash 0\right\}\right) \times \Sigma_{l_{1}} l_{1}(w B), \\
p=(r, x) \mapsto\left(t_{p} \psi_{1}\left(\frac{r}{|r|_{l_{1}}}\right), t_{p} b_{1}^{\prime}\left(\frac{x}{|r|_{l_{1}}}\right), t_{p} \psi_{2}\left(\frac{r}{|r|_{l_{1}}}\right), t_{p}^{\prime} b_{2}^{\prime}\left(\frac{x}{\mid \gamma_{l_{1}}}\right)\right)
\end{gathered}
$$

where

$$
t_{p}=\frac{|r|_{l_{1}}+|x|_{w}}{1+\left|b_{1}^{\prime}\left(x /|\gamma|_{l_{1}}\right)\right|_{B}+\left|b_{2}^{\prime}\left(x / \mid \gamma_{l_{1}}\right)\right|_{w}}
$$

Now $b$ is continuous since it is coordinate-wise continuous. (Note that $\mid \lambda_{l_{1}}$ cannot be zero.)

$$
\begin{aligned}
&\left.\left.b^{-1}: \Sigma_{l_{1}}\left(l_{1} \backslash\{0\}\right) \times \Sigma_{l_{1}} B \times \Sigma_{l_{1}}\left(l_{1} \backslash 0\right\}\right) \times \Sigma_{l_{1}} l_{1}(w B) \rightarrow \Sigma_{l_{1}}\left(l_{1} \backslash 0\right\}\right) \times \Sigma_{l_{1}} l_{1}(w B) \\
& q:(r, y, s, z) \mapsto\left(t_{q} \psi^{-1}\left(\frac{r}{\left.\left|H_{l_{1}}+\right| s\right|_{l_{1}}}, \frac{s}{\left.\left|H_{l_{1}}+\right| s\right|_{l_{1}}}\right),\right. \\
&\left.{ }^{t} b^{\prime-1}\left(\frac{y}{\left.\left|I_{l_{1}}+\right| s\right|_{l_{1}}}, \frac{z}{|r|_{l_{1}}+|s|_{l_{1}}}\right)\right)
\end{aligned}
$$

where 


$$
t_{q}=\frac{|r|_{l_{1}}+|y|_{B}+|s|_{l_{1}}+|z|_{w}}{1+\left|b^{\prime-1}\left(y /\left(|r|_{l_{1}}+|s|_{l_{1}}\right), z /\left(|r|_{l_{1}}+|s|_{l_{1}}\right)\right)\right|_{w}} .
$$

The reader may now show that $b$ is the required homeomorphism. Remember that $g^{-1}\left(\Sigma_{l_{1}} B \times\{0\}\right)$ is radial. Also, look at the diagram above. When $x \in \Sigma_{l_{1}} l_{1}(w B)$ and $\phi(x)$ has second coordinate zero, then $b_{2}^{\prime} \circ b^{\prime}(x)$ will be zero. This will give us condition (2).

A closed set $K \subset B$ has property $Z$ (is a $Z$-set) in $B$ if for each nonempty, homotopically trivial, open set $U$ in $B$ it is true that $U \backslash K$ is nonempty and homotopically trivial.

We will need the following theorem concerning $Z$-sets:

Theorem 4. Given a Banacb space $B \cong B^{\omega}$, then a countable union of $Z$. sets $\bigcup_{i=1}^{\infty} K^{i}$ is negligible in $B$, i.e. $B \cong B \backslash \bigcup_{i=1}^{\infty} K^{i}$.

Proof. This result is due to Chapman and Torunczyk, independently. See [2] or [14].

Remark 2. $\Sigma_{l_{1}}\left(l_{1} \backslash\{0\}\right) \cong \Sigma_{l_{1}} l_{1}=l_{1}$. This is due to the fact that $K_{n}=$ $\left\{\left\{x_{i}\right\} \in \Sigma_{l_{1}} l_{1} \mid x_{n}=0\right\}$ is a $Z$-set in $\Sigma_{l_{1}} l_{1}$ and $\left(\Sigma_{l_{1}} l_{1}\right) \backslash\left(\bigcup_{n=1}^{\infty} K_{n}\right)=\Sigma_{l_{1}}\left(l_{1} \backslash(0\}\right)$. See Cutler [3, Theorem 1] for a proof that $\left(\Sigma_{l_{1}} l_{1}\right) \backslash\left(\bigcup_{n=1}^{\infty} K_{n}\right)$ is homeomorphic with $\Sigma_{l_{1}} l_{1} . \Sigma_{l_{1}}\left(\Sigma_{l_{1}}\left(l_{1} \backslash\{0\}\right)\right) \cong l_{1}$ by a similar proof.

\section{Main results.}

Lemma 5. Any infinite-dimensional Banach space $B \cong B^{\omega}$ is bomeomorphic to $l_{1}(w B)$.

Proof. Let $b$ be the homeomorphism guaranteed by Lemma 3,

$$
b: \Sigma_{l_{1}}\left(l_{1} \backslash\{0\}\right) \times \Sigma_{l_{1}} l_{1}(w B) \rightarrow \Sigma_{l_{1}}\left(l_{1} \backslash\{0\}\right) \times \Sigma_{l_{1}} B \times \Sigma_{l_{1}}\left(l_{1} \backslash\{0\}\right) \times \Sigma_{l_{1}} l_{1}(w B),
$$

and let $b_{i}$ be the projection of $b$ onto the $i$ th coordinate, $i=1,2,3,4$. Define

and define

$$
\Sigma_{l_{1}^{f}}\left(\Sigma_{l_{1}} B\right)=\left\{\left\{x_{i}\right\} \in \Sigma_{l_{1}}\left(\Sigma_{l_{1}} B\right) \mid x_{i}=0 \text { for almost all } i\right\},
$$

$$
\begin{aligned}
& A=\left\{(r, x) \in \Sigma_{l_{1}}\left(l_{1} \backslash\{0\}\right) \times \Sigma_{l_{1}} l_{1}(w B) \mid\right. \\
&b_{4} \circ \underbrace{\left(b_{3}, b_{4}\right) \circ \ldots \circ\left(b_{3}, b_{4}\right)}_{n-1}(r, x)=0 \text { for some } n=1,2, \ldots\}
\end{aligned}
$$

where 


$$
\begin{aligned}
\left(b_{3}, b_{4}\right): \Sigma_{l_{1}}\left(l_{1} \backslash\{0\}\right) \times \Sigma_{l_{1}} l_{1}(w B) & \left.\rightarrow \Sigma_{l_{1}}\left(l_{1} \backslash 0\right\}\right) \times \Sigma_{l_{1}} l_{1}(w B), \\
(r, x) & \mapsto\left(b_{3}(r, x), b_{4}(r, x)\right) .
\end{aligned}
$$

We will let $\left(b_{3}, b_{4}\right)^{n}$ denote the $n$-fold composition of $\left(b_{3}, b_{4}\right)$. (Note that $A$ is a set of the form $\Sigma_{l_{1}}\left(l_{1} \backslash\{0\}\right) \times N$, i.e. $A$ does not depend on the first coordinate.) Then define

$$
\begin{aligned}
& H: A \rightarrow \Sigma_{l_{1}}\left(\Sigma_{l_{1}}\left(l_{1} \backslash\{0\}\right)\right) \times \Sigma_{l_{1}^{f}}\left(\Sigma_{l_{1}} B\right), \\
& (r, x) \mapsto\left(\left\{b_{1} \circ\left(b_{3}, b_{4}\right)^{n-1}(r, x)\right\}_{n},\left\{b_{2} \circ\left(b_{3}, b_{4}\right)^{n-1}(r, x)\right\}_{n}\right) .
\end{aligned}
$$

$H$ is into by condition (1) of Lemma 3. Given $(r, x) \in A$, there exists an $n$ such that $b_{4} \circ\left(b_{3}, b_{4}\right)^{n}(r, x)=0$. Then let $s=b_{3} \circ\left(b_{3}, b_{4}\right)^{n}(r, x)$. By condition (1), $b(s, 0)=\left(\psi_{1}(s), 0, \psi_{2}(s), 0\right)$. But $\psi$ is an isomorphism, and therefore, $H(r, x)$ is summable. In fact, $|H(r, x)|_{B}=|(r, x)|_{A}$ where ||$_{A}$ is the norm on $A$ and ||$_{B}$

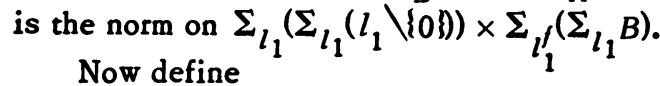

$$
\begin{aligned}
1_{b}= & b=\left(b_{1}, b_{2}, b_{3}, b_{4}\right) \\
2^{2} b= & \left(b_{1}, b_{2}, b \circ\left(b_{3}, b_{4}\right)\right) \\
\vdots & \\
{ }^{n} b= & \left(b_{1}, b_{2}, b_{1} \circ\left(b_{3}, b_{4}\right), b_{2} \circ\left(b_{3}, b_{4}\right), \cdots,\right. \\
& \left.b_{1} \circ\left(b_{3}, b_{4}\right)^{n-2}, b_{2} \circ\left(b_{3}, b_{4}\right)^{n-2}, b \circ\left(b_{3}, b_{4}\right)^{n-1}\right) .
\end{aligned}
$$

${ }^{n} b: \Sigma_{l_{1}}\left(l_{1} \backslash\{0\}\right) \times \Sigma_{l_{1}} l_{1}(w B) \rightarrow \underbrace{\left.\left[\left(\Sigma_{l_{1}}\left(l_{1} \backslash\{0\}\right) \times \Sigma_{l_{1} B} B\right) \times \cdots \times\left(\Sigma_{l_{1}}\left(l_{1} \backslash 0\right\}\right) \times \Sigma_{l_{1} B} B\right)\right]}_{n-2}$

$$
\times \Sigma_{l_{1}}\left(l_{1} \backslash\{0\}\right) \times \Sigma_{l_{1}} B \times \Sigma_{l_{1}}\left(l_{1} \backslash\{0\}\right) \times \Sigma_{l_{1}} l_{1}(w B) .
$$

${ }^{n} b$ is a homeomorphism for each $n$ and ${ }^{n} b$ is distance preserving from zero by condition 3 of Lemma 3.

To see that $H$ is continuous, given $\left\{\left(r^{j}, x^{j}\right)\right\}_{j}$ converging to $(r, x)$ in $A$ and $\epsilon>0$, pick $N$ such that

$$
\sum_{i=N+1}^{\infty}\left[\left|b_{1} \circ\left(b_{3}, b_{4}\right)^{i-1}(r, x)\right|+\left|b_{2} \circ\left(b_{3}, b_{4}\right)^{i-1}(r, x)\right|\right]<\epsilon / 8 .
$$

Now since ${ }^{N+1} h$ is a homeomorphism, given $\epsilon / 8$ there exists a $J$ such that $j>J$ implies 


$$
\begin{aligned}
& \sum_{i=1}^{N}\left|b_{1} \circ\left(b_{3}, b_{4}\right)^{i-1}(r, x)-b_{1} \circ\left(b_{3}, b_{4}\right)^{i-1}\left(r^{j}, x^{j}\right)\right| \\
& \quad+\sum_{i=1}^{N}\left|b_{2} \circ\left(b_{3}, b_{4}\right)^{i-1}(r, x)-b_{2} \circ\left(b_{3}, b_{4}\right)^{i-1}\left(r^{j}, x^{j}\right)\right| \\
& +\left|b_{3} \circ\left(b_{3}, b_{4}\right)^{N}(r, x)-b_{3} \circ\left(b_{3}, b_{4}\right)^{N}\left(r^{j}, x^{j}\right)\right| \\
& +\left|b_{4} \circ\left(b_{3}, b_{4}\right)^{N}(r, x)-b_{4} \circ\left(b_{3}, b_{4}\right)^{N}\left(r^{j}, x^{j}\right)\right|<\frac{\epsilon}{8} .
\end{aligned}
$$

In particular, the last two terms in the four term sum are less than $\epsilon / 8$. Since $H$ is distance preserving from zero, $\left|H(r, x)-H\left(r^{j}, x^{j}\right)\right|<\epsilon$. To see this, note that

$$
\left|b_{3} \circ\left(b_{3}, b_{4}\right)^{N}(r, x)-b_{3} \circ\left(b_{3}, b_{4}\right)^{N}\left(r^{j}, x^{j}\right)\right|<\frac{\epsilon}{8}
$$

says that $\left|b_{3} \circ\left(b_{3}, b_{4}\right)^{N}\left(r^{j}, x^{j}\right)\right|<\epsilon / 8+\epsilon / 8$ since

$$
\begin{aligned}
\mid b_{3} & \circ\left(b_{3}, b_{4}\right)^{N}(r, x)|+| b_{4} \circ\left(b_{3}, b_{4}\right)^{N}(r, x) \mid \\
& =\sum_{i=N+1}^{\infty}\left[\left|b_{1} \circ\left(b_{3}, b_{4}\right)^{i-1}(r, x)\right|+\left|b_{2} \circ\left(b_{3}, b_{4}\right)^{i-1}(r, x)\right|\right] .
\end{aligned}
$$

The same holds for $\left|b_{4} \circ\left(b_{3}, b_{4}\right)^{N}\left(r^{j}, x^{j}\right)\right| . H$ is clearly one-to-one and onto.

To see that $H^{-1}$ is continuous, let $\left\{\left(r^{j}, x^{j}\right)\right\}$ converge to $(r, x)$ in $\Sigma_{l_{1}}\left(\Sigma_{l_{1}}\left(l_{1} \backslash\{0\}\right)\right) \times \Sigma_{l_{1}}\left(\Sigma_{l_{1}} B\right)$ and suppose we are given $\epsilon>0$. Let $N_{0}^{\prime}$ be a positive integer such that $x_{n}=0$ for $n \geq N_{0}^{\prime}$ where $x_{n}$ is the $n$th coordinate in $x=\left(x_{i}\right)$. Then pick $N_{0} \geq N_{0}^{\prime}$ so that $\Sigma_{N_{0}+1}^{\infty}\left(\left|r_{i}\right|+\left|x_{i}\right|\right)<1$. Then, let $(s, 0)$ with $|s|<1$ be the last two coordinates in $N_{0} b\left(H^{-1}(r, x)\right)$. Similarly, let $\left(s^{j}, y^{j}\right)$ be the last two coordinates in ${ }^{N} b_{b}\left(H^{-1}\left(r^{j}, x^{j}\right)\right)$. Next, pick $J$ such that $j \geq J$ implies $\left|s^{j}\right|<1$. Now, ${ }^{N_{0}} b$ is a homeomorphism. Therefore, given ${ }^{N_{0}} b\left(H^{-1}(r, x)\right)$ and $\epsilon>0$ there is a $\delta>0$ such that if a point is within $\delta$ of ${ }^{N_{0}} b\left(H^{-1}(r, x)\right)$ then its image under $\left({ }^{N}{ }_{b}\right)-1$ is within $\epsilon$ of $H^{-1}(r, x)$.

Next, pick $N>N_{0}$ so that $\Sigma_{N+1}^{\infty}\left|\psi_{1} \circ \psi_{2} \circ \ldots \circ \psi_{2}(s)\right|<\delta / 16$. Then, let $\delta_{0}=\delta / 4 N^{2}$. Given $\delta_{0}$, pick $\eta \leq \delta_{0}$ such that $z \in \Sigma_{l_{1}} l_{1}(w B)$ and $|z-0|_{w}<\eta$ implies

$$
\left|b_{1}^{\prime}(z)-0\right|_{B}+\left|b_{2}^{\prime}(z)-0\right|_{w}<\delta_{0}
$$

Then pick $J_{0}^{\prime} \geq J$ such that $j>J_{0}^{\prime}$ implies

$$
\frac{\left|y^{j}\right|}{\left|s^{j}\right|}<\eta, \frac{\left|b_{4} \circ\left(b_{3}, b_{4}\right)^{n-1}\left(s^{j}, y^{j}\right)\right|}{\left|b_{3} \circ\left(b_{3}, b_{4}\right)^{n-1}\left(s^{j}, y^{j}\right)\right|}<\eta, \text { for } n=1,2, \cdots, N \text {. }
$$

Then $j \geq J_{0}^{\prime}$ (looking at the definition of $b$ ) 


$$
\begin{aligned}
& \left|\frac{\left(\left|s^{j}\right|+\left|y^{j}\right|\right) \psi_{1}\left(s^{j} /\left|s^{j}\right|\right)}{1+\left|b_{1}^{\prime}\left(y^{j} /\left|s^{j}\right|\right)\right|+\left|b_{2}^{\prime}\left(y^{j} /\left|s^{j}\right|\right)\right|}-\psi_{1}\left(s^{j}\right)\right| \\
& \quad=\left|\frac{\left(\left|s^{j}\right|+\left|y^{j}\right|\right) \psi_{1}\left(s^{j} /\left|s^{j}\right|\right)}{1+\left|b_{1}^{\prime}\left(y^{j} /\left|s^{j}\right|\right)\right|+\left|b_{2}^{\prime}\left(y^{j} /\left|s^{j}\right|\right)\right|}-\right| s^{j}\left|\psi_{1}\left(s^{j} /\left|s^{j}\right|\right)\right| \\
& \quad \leq\left|\frac{\left|s^{j}\right|+\left|y^{j}\right|-\left|s^{j}\right|-\left|s^{j}\right|\left(\left|b_{1}^{\prime}\left(y^{j} /\left|s^{j}\right|\right)\right|+\left|b_{2}^{\prime}\left(y^{j} /\left|s^{j}\right|\right)\right|\right) \mid}{1+\left|b_{1}^{\prime}\left(y^{j} /\left|s^{j}\right|\right)\right|+\left|b_{2}^{\prime}\left(y^{j} /\left|s^{j}\right|\right)\right|}\right| \\
& \quad \leq|| y^{j}|-| s^{j}\left|\left(\left|b_{1}^{\prime}\left(y^{j} /\left|s^{j}\right|\right)\right|+\left|b_{2}^{\prime}\left(y^{j} /\left|s^{j}\right|\right)\right|\right)\right| \leq \delta_{0}=\delta / 4 N^{2} .
\end{aligned}
$$

Similarly

$$
\left|\frac{\left(\left|s^{j}\right|+\left|y^{j}\right|\right) \psi_{2}\left(s^{j} /\left|s^{j}\right|\right)}{1+\left|b_{1}^{\prime}\left(y^{i} /\left|s^{j}\right|\right)\right|+\left|b_{2}^{\prime}\left(y^{j} /\left|s^{j}\right|\right)\right|}-\psi_{2}\left(s^{j}\right)\right| \leq \delta_{0} .
$$

Also, let

$$
p=\frac{\left(\left|s^{j}\right|+\left|y^{j}\right|\right) \psi_{2}\left(s^{j} /\left|s^{j}\right|\right)}{1+\left|b_{1}^{\prime}\left(y^{j} /\left|s^{j}\right|\right)\right|+\left|b_{2}^{\prime}\left(y^{i} /\left|s^{j}\right|\right)\right|}=b_{3}\left(s^{j}, y^{j}\right), .
$$

and let $q=b_{4}\left(s^{j}, y^{j}\right)$. Then, by the same argument as above

$$
\left|\frac{(|p|+|q|) \psi_{1}(p /|p|)}{1+\left|b_{1}^{\prime}(q /|p|)\right|+\left|b_{2}^{\prime}(q /|p|)\right|}-\psi_{1}(p)\right| \leq \| q|-| p\left|\left(\left|b_{1}^{\prime}(q /|p|)\right|+\left|b_{2}^{\prime}(q /|p|)\right|\right)\right| \leq \delta_{0} \text {. }
$$

But $\left|\psi_{1} \circ \psi_{2}\left(s^{j}\right)-\psi_{1}(p)\right| \leq\left|\psi_{2}\left(s^{j}\right)-p\right|<\delta_{0}=\delta / 4 N^{2}$ and $\psi$ is an isomorphism. Therefore

$$
\begin{aligned}
& \left|\psi_{1} \circ \psi_{2}\left(s^{\prime}\right)-\frac{(|p|+|q|) \psi_{1}(p /|p|)}{1+\left|b_{1}^{\prime}(q /|p|)\right|+\left|b_{2}^{\prime}(q /|p|)\right|}\right| \\
& \quad \leq\left|\psi_{1} \circ \psi_{2}\left(s^{\prime}\right)-\psi_{1}(p)\right|+\left|\frac{(|p|+|q|) \psi_{1}(p /|p|)}{1+\left|b_{1}^{\prime}(q /|p|)\right|+\left|b_{2}^{\prime}(q /|p|)\right|}-\psi_{1}(p)\right| \leq 2 \delta / 4 N^{2} .
\end{aligned}
$$

By induction, the $k$ th coordinate for $k \leq N$ satisfies

$$
\left|\psi_{1} \circ \psi_{2}^{k-1}\left(s^{j}\right)-b_{1} \circ\left(b_{3}, b_{4}\right)^{k-1}\left(s^{j}, y^{j}\right)\right|<k \delta / 4 N^{2} .
$$

Here $\psi_{2}^{k-1}$ denotes the $(k-1)$-fold composition of $\psi_{2}$. But now pick $J_{0} \geq J_{0}^{\prime}$ such that for $j>J_{0}$ 
(1) $\left|\psi_{1} \circ \psi_{2}^{k-1}(s)-b_{1} \circ\left(b_{3}, b_{4}\right)^{k-1}\left(s^{j}, y^{j}\right)\right|<\delta / 4 N$ for $k=1,2, \cdots, N$;

(2) $\left|y^{j}\right|<\delta / 4$; and

(3) $\left\|\left(s^{j}, y^{j}\right)|-|(s, 0)\right\|<\delta / 16$.

Then

$$
\begin{aligned}
\left|s^{i}-s\right|+\left|y^{j}-0\right|= & \sum_{i=1}^{N}\left|\psi_{1} \circ \psi_{2}^{i-1}(s)-\psi_{1} \circ \psi_{2}^{i-1}\left(s^{j}\right)\right|+\left|y^{j}\right| \\
& +\sum_{i=N+1}^{\infty}\left|\psi_{1} \circ \psi_{2}^{i-1}(s)-\psi_{1} \circ \psi_{2}^{i-1}\left(s^{j}\right)\right| \\
\leq & \sum_{i=1}^{N}\left|\psi_{1} \circ \psi_{2}^{i-1}(s)-b_{1} \circ\left(b_{3^{\prime}}, b_{4}\right)^{i-1}\left(s^{j}, y^{j}\right)\right| \\
& +\sum_{i=1}^{N}\left|b_{1} \circ\left(b_{3}, b_{4}\right)^{i-1}\left(s^{j}, y^{j}\right)-\psi_{1} \circ \psi_{2}^{i-1}\left(s^{j}\right)\right| \\
& +\frac{\delta}{4}+\sum_{i=N+1}^{\infty}\left|\psi_{1} \circ \psi_{2}^{i-1}(s)\right|+\sum_{i=N+1}^{\infty}\left|\psi_{1} \circ \psi_{2}^{i-1}\left(s^{j}\right)\right| \\
\leq & N(\delta / 4 N)+N(\delta / 4 N)+\delta / 4+\delta / 16+(\delta / 16+\delta / 16) \\
= & 15 \delta / 16 .
\end{aligned}
$$

Therefore, pick $J_{1} \geq J_{0}$ such that $j \geq J_{1}$ implies

$$
\left|\pi_{N_{0}} \circ\left[{ }^{N_{0}} b\left(H^{-1}(r, x)\right)\right]-\pi_{N_{0}} \circ\left[{ }^{N_{0}} b\left(H^{-1}\left(r^{j}, x^{j}\right)\right)\right]\right|<\delta / 16,
$$

where $\pi_{N_{0}}$ is projection onto the first $N_{0}$ coordinates. Then $\mid H^{-1}(r, x)-$ $H^{-1}\left(r^{j}, x^{j}\right) \mid<\epsilon$ whenever $j \geq J_{1}$. Thus $H^{-1}$ is continuous and $H$ is a homeomorphism.

By Remark 2, $\Sigma_{l_{1}}\left(l_{1} \backslash\{0\}\right) \cong l_{1}$ and $\Sigma_{l_{1}}\left(\Sigma_{l_{1}}\left(l_{1} \backslash\{0\}\right)\right) \cong l_{1}$. Using the fact that $A=\Sigma_{l_{1}}\left(l_{1} \backslash\{0\}\right) \times N$ for some $N \subset \Sigma_{l_{1}} l_{1}(w B)$, we may change $H$ to a homeomorphism $H^{\prime}$,

$$
H^{\prime}: l_{1} \times N \rightarrow l_{1} \times \Sigma_{l_{1}^{f}}\left(\Sigma_{l_{1}} B\right)
$$

$H^{\prime}$ may be extended to $G_{\delta}$ subsets of $l_{1} \times \Sigma_{l_{1}} l_{1}(w B)$ and $l_{1} \times \Sigma_{l_{1}}\left(\Sigma_{l_{1}} B\right)$ by a theorem of Lavrentiev (see Kuratowski [9, p. 429]). But these $G_{\delta}$ sets are dense in the respective spaces, and the complements are countable unions of Z-sets. They are $Z$-sets since we may leave the $l_{1}$ coordinates alone and use the fact 
that $l_{1} \times \Sigma_{l_{1}} l_{1}(w B)$ and $l_{1} \times \Sigma_{l_{1}^{f}}\left(\Sigma_{l_{1}} B\right)$ are contained in the respective $G_{\delta}$ 's. Then given a closed set $K$ in the complement of, say, $l_{1} \times \Sigma_{l_{1}^{f}} l_{1}(w B)$ and a map $f: S^{n} \rightarrow \mathcal{U} \backslash K$, cover the image of $S^{n}$ under $f$, a compact set, by a finite number of convex open sets contained in $\mathcal{U} \backslash K$. Then pick an $M$ such that $\pi_{l_{1}}\left(f\left(s^{n}\right)\right) \times$ $\left[\pi_{M}\left(\pi_{w} f\left(S^{n}\right)\right) \times\{0\}\right]$ is contained in the union of the open sets. Here $\pi_{M}$ is projection onto the first $M$ coordinates and $\pi_{w}$ is projection onto $\Sigma_{l_{1}} l_{1}(w B)$. Now, straight line homotopy each point of $f\left(S^{n}\right)$ to its corresponding point with $\{0\}$ from $M+1$ on in the $\Sigma_{l_{1}} l_{1}(w B)$ factor. This gives an extension of $f$ to a function $\bar{f}: E^{n+1} \rightarrow \mathcal{U} \backslash K$. Hence $K$ is a $Z$-set.

Finally, by Theorem $4, l_{1} \times \Sigma_{l_{1}} l_{1}(w B) \cong l_{1} \times \Sigma_{l_{1}}\left(\Sigma_{l_{1}}(B)\right)$. But $l_{1} \times$ $\Sigma_{l_{1}} l_{1}(w B) \cong l_{1}(w B)$ since $\Sigma_{l_{1}} l_{1}(w B)=l_{1}(w B)$ and $l_{1}(w B) \cong l_{1} \times l_{1}(w B)$. Similarly $l_{1} \times \Sigma_{l_{1}}\left(\Sigma_{l_{1}} B\right) \cong \Sigma_{l_{1}} B$. By a theorem of the author's. [12]. $\Sigma_{l_{1}} B \cong B$. Therefore, since $B \cong B^{\omega}$ by assumption, $B \cong l_{1}(w B)$.

To extend this result to Fréchet spaces, we need the following obvious lemma:

Lemma 6. Let $\left\{||_{i}\right\}$ be a collection of pseudo-norms which determine the topology of the Fréchet space $F$. Then there is, for each $i$, a continuous linear surjection $T_{i}$ of $F$ onto the Banach space $F /||_{i}=B_{i}$.

Theorem 7. Any infinite-dimensional Fréchet space $F \cong F^{\omega}$ is bomeomorphic with $l_{2}(w F)$.

Proof. By Lemma 6, there are continuous linear surjections $T_{i}: F \rightarrow B_{i}$. By a result in [1], this gives us that $F \cong B_{i} \times N_{i}$ where $N_{i}$ is the kernel of $T_{i}$. Therefore, since $F \cong F^{\omega}$,

$$
F \simeq B_{i} \times N_{i} \simeq\left(B_{i} \times N_{i}\right)^{\omega} \simeq B_{i}^{\omega} \times\left(B_{i} \times N_{i}\right)^{\omega} \simeq B_{i}^{\omega} \times F .
$$

Therefore,

$$
F^{\omega} \simeq \prod_{i=1}^{\infty}\left(B_{i}^{\omega} \times F\right) \cong\left(\prod_{i=1}^{\infty} B_{i}^{\omega}\right) \times F .
$$

By a theorem of the author's [12],

$$
B_{i}^{\omega} \cong \Sigma_{l_{1}} B_{i} \text { and } \prod_{i=1}^{\infty} \Sigma_{l_{1}} B_{i} \cong \Sigma_{l_{1}}\left(\Sigma_{l_{1}} B_{i}\right)_{i} \text {. }
$$

Therefore, $F^{\omega} \cong \Sigma_{l_{1}}\left(\Sigma_{l_{1}} B_{i}\right)_{i} \times F$. But $\Sigma_{l_{1}}\left(\Sigma_{l_{1}} B_{i}\right)_{i}$ is a Banach space with weight equal to the weight of $F$, and it is homeomorphic with its countable product. Therefore, $\Sigma_{l_{1}}\left(\Sigma_{l_{1}} B_{i}\right)_{i} \cong l_{1}(w F)$. But then $F \cong l_{1}(w F) \times F$, and Theorem 1 gives $l_{1}(w F) \cong l_{1}(w F) \times F$. Thus, $F \cong l_{1}(w F)$. But $l_{1}(w F)$ is homeomorphic with $l_{2}(w F)$. (See Mazur, [10].) Therefore, $F \cong l_{2}(w F)$. 


\section{REFERENCES}

1. C. Bessaga, On topological classification of complete linear metric spaces, Fund. Math. 56 (1964/65), 251-288. MR 31 \#2580.

2. T. A. Chapman, Deficiency in infinite-dimensional manifolds, General Topology and Appl. 1 (1971), 263-272.

3. W. H. Cutler, Negligible subsets of infinite-dimensional Fréchet manifolds, Proc. Amer. Math. Soc. 23 (1969), 668-675. MR 40 \#2133.

4. D. W. Henderson, Stable classification of infinite-dimensional manifolds by homotopy-type, Invent. Math. 12 (1971), 48-56. MR 44 \#7594.

5. - Corrections and extensions of two papers about infinite-dimensional manifolds, General Topology and Appl. 1 (1971), 321-327. MR 45 \#2754.

6. V. L. Klee, Convex bodies and periodic homeomorphisms in Hilbert space, Trans. Amer. Math. Soc. 74 (1953), 10-43. MR 14, 989.

7. - A note on topological properties of normed linear spaces, Proc. Amer. Math. Soc. 7 (1956), 673-674. MR 17, 1227.

8. - Some topological properties of convex sets, Trans. Amer. Math. Soc. 78 (1955), 30-45. MR 16, 1030.

9. K. Kuratowski, Topologie. Vol. I, 2nd ed., Monografie Mat., Tom 20, WarszawaWrocław, 1948; English transl., New ed., rev. and aug., Academic Press, New York; PWN, Warsaw, 1966. MR 10, 389; 36 \#840.

10. S. Mazur, Une remarque sur l'homéomorphie des champs fonctionels, Studia Math. 1 (1929), 83-85.

11. E. Michael, Local properties of topological spaces, Duke Math. J. 21 (1954), 163171. MR 15, 977.

12. W. E. Terry, Conditions for a TVS to be homeomorphic with its countable product, Trans. Amer. Math. Soc. 190 (1974), 233-242.

13. H. Toruńczyk, Absolute retracts as factors of normed linear spaces (to appear).

14. - , $(G, K)$-absorbing and skeletonized sets in metric spaces, Dissertationes Math. (to appear).

DEPARTMENT OF MATHEMATICS, CORNELL UNIVERSITY, ITHACA, NEW YORK 14850

Current address: Department of Mathematics, University of Saskatchewan, Saskatoon, Saskatchewan S7N 0w0 Canada 